OPEN ACCESS

Edited by:

Saverio Marchi,

Marche Polytechnic University, Italy

Reviewed by:

Anca Maria Cimpean

Victor Babes University of Medicine

and Pharmacy, Romania

Prasanna Ekambaram,

University of Pittsburgh, United States

*Correspondence:

Shuli Zhao

shulizhao79@163.com

Jian-hong Shi

shijianhong@hbu.edu.cn

†These authors have contributed equally to this work

Specialty section

This article was submitted to Molecular and Cellular Oncology,

a section of the journal

Frontiers in Oncology

Received: 10 April 2019

Accepted: 20 August 2019

Published: 11 September 2019

Citation:

Lin D, Shen Y, Qiao S, Liu W, Zheng L,

Wang Y, Cui N, Wang Y, Zhao S and

Shi J (2019) Upregulation of OTUD7B

(Cezanne) Promotes Tumor

Progression via AKTNEGF Pathway in

Lung Squamous Carcinoma and Adenocarcinoma. Front. Oncol. 9:862.

doi: 10.3389/fonc.2019.00862

\section{Upregulation of OTUD7B (Cezanne) Promotes Tumor Progression via AKT/VEGF Pathway in Lung Squamous Carcinoma and Adenocarcinoma}

\author{
Dan-dan Lin ${ }^{1+}$, Yang Shen ${ }^{1+}$, Shu Qiao ${ }^{2}$, Wen-wen Liu ${ }^{1}$, Lishuang Zheng ${ }^{1}$, Ya-nan Wang ${ }^{3}$, \\ Naipeng Cui ${ }^{2}$, Yun-fan Wang ${ }^{1}$, Shuli Zhao ${ }^{4 *}$ and Jian-hong Shi ${ }^{1 *}$ \\ ${ }^{1}$ Central Laboratory, Hebei Key Laboratory of Cancer Radiotherapy and Chemotherapy, Affiliated Hospital of Hebei \\ University, Baoding, China, ${ }^{2}$ Department of Breast Surgery, Affiliated Hospital of Hebei University, Baoding, China, \\ ${ }^{3}$ Department of Pathology, Affiliated Hospital of Hebei University, Baoding, China, ${ }^{4}$ General Clinical Research Center, Nanjing \\ First Hospital, Nanjing Medical University, Nanjing, China
}

OTUD7B, a multifunctional deubiquitinylase, plays an essential role in inflammation and proliferation signals. However, its function in lung cancer remains largely unknown. The aim of this study was to evaluate the prognostic significance of OTUD7B in patients with lung adenocarcinoma and squamous carcinoma and to characterize its molecular mechanisms in lung cancer progression and metastasis. Two tissue microarrays containing 150 pairs of lung squamous carcinoma and matched adjacent non-cancer tissues, and one tissue microarray containing 75 pairs of lung adenocarcinoma and adjacent non-cancer tissues were included, and immunohistochemical staining was performed to assess the clinical relevance of OTUD7B in non-small cell lung cancer. OTUD7B is highly expressed in both lung squamous carcinoma and adenocarcinoma and correlates with a worse prognosis. MTT proliferation, colony formation, migration and invasion assays and immunoblotting assay in $\mathrm{NCl}-\mathrm{H} 358$ and $\mathrm{A} 549$ cell lines suggested that OTUD7B enhances EGF-induced Akt signal transduction and promotes lung cancer cell proliferation and migration. Immunohistochemical staining of large-scale lung cancer subjects (171 cases) revealed positive correlation of OTUD7B and VEGF expression. ELISA and tube formation assay revealed OTUD7B promotes VEGF production and angiogenesis. $\mathrm{NCl}-\mathrm{H} 358$ tumor model demonstrated OTUD7B is required for lung tumor progression by facilitating activation of Akt signaling. These findings collectively identified OTUD7B as an independent predictive factor for the prognosis of non-small cell lung cancer and revealed OTUD7B promotes lung cancer cell proliferation and metastasis via Akt/VEGF signal pathway.

Keywords: OTUD7B, lung squamous carcinoma, lung adenocarcinoma, prognosis, VEGF, Akt 


\section{INTRODUCTION}

Lung cancer is one of the most common cancers and leading causes of cancer-related deaths worldwide. Most cases of lung cancer are non-small cell lung cancer (NSCLC). NSCLC can be further sorted into several types, including lung squamous carcinoma (LUSC) and lung adenocarcinoma (LAD), which are the most commonly diagnosed histological subtypes of NSCLC (1). High incidence of post-chemotherapy metastasis and recurrence generates challenge as for its resistance to conventional systemic radiotherapy and chemotherapy (2). The importance of understanding the molecular biology of LUSC and LAD has recently gained considerable attention.

Ubiquitination is an important mechanism that regulates cancer progression $(3,4)$. Deubiquitylases (DUBs) are enzymes that hydrolysis ubiquitin chains from target proteins and contribute to their stabilization and activation $(5,6)$. DUBs play essential roles in inflammation and proliferation signal transduction and are considered effective therapeutic targets against lung cancer. OTUD7B, a DUB belonging to A20 subgroup of ovarian tumor (OUT) protein superfamily, functions as an inflammation and NF- $\mathrm{B}$ signaling regulator (7-11). Accumulate data suggested that OTUB7B appears to be the primary regulatory mechanism to growth signals, including activation of mTORC2/Akt pathway (12), regulation of E2F1dependent HIF $2 \alpha$ expression (13), stabilization of HIF1 $\alpha$ (14), and EGFR (15).

Although some evidence revealed the role of OTUD7B in cancer progression $(10,12,15-18)$, the molecular mechanisms of OTUD7B participation in invasion and metastasis in NSCLCs remains elusive. Here, we demonstrate that OTUD7B were predominantly upregulated in LUSC and LAD tissues compared with adjacent normal lung tissues. OTUD7B upregulation predicts an increased risk for cancer metastasis in LUSC and LAD patients. Moreover, we found that OTUD7B regulation in lung cancer progression associates with tumor angiogenesis via Akt/VEGF pathway.

\section{MATERIALS AND METHODS}

\section{Antibodies and Reagents}

Antibodies for OTUD7B (16605-1-AP) and JNK (10023-1-AP) were from Proteintech Group, Inc. (Chicago, USA). Antibody for GAPDH (AC033) was from Abclonal (Wuhan, China). Antibodies for p70S6K (2708), p-p70S6K T389 (9206), pp70S6K T421/S424 (9204), ERK1/2 (9102), p-ERK1/2 T202/Y204 (9101), p-Akt S473 (4060), p-p38 T180/Y182 (9215), and pJNK T183/Y185 (4668) were purchased from Cell Signaling Technology (Boston, USA). Antibody for p38 (35478) was from SAB. Antibody for Akt1(SC-5298) was from Santa Cruz Biotechnology (Dallas, USA). Human VEGFA monoclonal antibody (M808) and Biotin-labeled VEGFA polyclonal antibody were purchased from Thermo Fisher Scientific (Waltham, USA). EGF was from Peprotech (Suzhou, China).

\section{Plasmids and shRNAs}

The human OTUD7B cDNA (accession NM_020205.4) was cloned into the lentivirus vector pLenti-CMV-EGFP-3Flag
TABLE 1 | Patient characteristics.

\begin{tabular}{lc}
\hline Variable & No. of patients (\%) \\
\hline No. of NSCLC patients & $225(100)$ \\
No. of LUSC patients & $150(66.7)$ \\
No. of LAD patients & $75(33.3)$ \\
Age: Median [range] & $62[25-84]$ \\
Gender & \\
Female & $46(20.4)$ \\
Male & $179(79.6)$ \\
AJCC stage & \\
I & $81(45)$ \\
II & $54(30)$ \\
III & $44(24.4)$ \\
IV & $1(0.6)$ \\
Lymphatic metastasis & \\
Absent & $122(56.5)$ \\
Present & $94(43.5)$ \\
Distant metastasis & \\
Absent & $145(64.4)$ \\
Present & \\
Survival & $224(99.6)$ \\
Death & $1(0.4)$ \\
\hline
\end{tabular}

to create the GFP or GFP-OTUD7B expression vector, and the OTUD7B cDNA sequence was confirmed by sequencing. LV3 lentiviral vectors encoding shRNAs silencing OTUD7B or a non-silencing control shRNA were purchased from GenePharma (Suzhou, China). The sequences of OTUD7B shRNAs: shOTUD7B\#1: TTCTCCGAACGTGTCACGT; shOTUD7B\#2: GCTGCGGAAAGCTTTGTATGC.

\section{Tissue Array and Immunohistochemical Staining}

Three tissue microarrays containing 225 pairs of lung cancer and corresponding non-cancer tissues with survival times were purchased from BioChip (Shanghai, China), of which 150 cases are LUSC and 75 cases are LAD. These samples were collected from 2004 to 2009. The study was approved by Institutional Review Board of Hebei University Affiliated Hospital. All of the patients provide informed consent. The immunohistochemistry assay and the analysis of clinicopathological features were based on 75 cases of LAD and 150 cases of LUSC from tissue microarray, excluded a few cases because of missing data. Detailed clinical and pathologic information of patients are displayed in Table 1.

We performed immunohistochemical (IHC) staining for OTUD7B and VEGF on the same paraffin embedded tissue blocks that were used for clinical diagnosis. Immunohistochemistry was performed using the avidin-biotin complex method, including heat-induced antigen-retrieval procedures. Incubation with polyclonal antibodies against OTUD7B (16605-1-AP; 1:1,000 dilution; Proteintech), VEGF (19003-1-AP; 1:500 dilution; Proteintech) was performed 
at $4^{\circ} \mathrm{C}$ overnight followed by incubation with horseradishperoxidase (HRP)-conjungated secondary antibody kit. Staining was assessed by pathologists who were blinded to the sample origins and the patient outcomes. Each sample was assigned a score according to the intensity of the nucleic, cytoplasmic and membrane staining (no staining $=0$; weak staining $=1$, moderate staining $=2$, and strong staining $=3$ ) and the extent of stained cells $(0-4=0,5-25=1,26-50=2,51-75=3$, and $76-100=4)$. Immunostaining score formula $=$ score of positive cell percentage $\times$ score of staining intensity. OTUD7B expression was qualified as low (IHC score 0-3), medium (IHC score 4-7), and high (IHC score 8-12).

\section{Gene Expression Databases}

Gene expression data were downloaded from public databases including Gene Expression Omnibus (GEO) and The Cancer Genome Atlas (TCGA). The cBioPortal for Cancer Genomics system (www.cbioportal.org) was used to analyze OTUD7B gene. R2: Genomics Analysis and Visualization Platform system (http://r2.amc.nl) was used to analyze the correlation between OTUD7B and VEGF.

\section{Cell Culture, Gene Silencing, and Overexpression}

Human NSCLC cell lines NCI-H358 and A549, human embryo kidney (HEK) 293 cell line and human endothelial EA.hy926 cell line were obtained from Cell Resource Center of Shanghai Institutes for Biological Sciences, Chinese Academy of Science, China. NCI-H358 and A549 cells were cultured in RPMI-1640 supplemented with 10\% FBS. HEK293 and EA.hy926 cells were maintained in Dulbecco's Modified Eagle's Medium with $10 \%$ FBS. All cell lines were cultured in a humidified atmosphere with $5 \% \mathrm{CO}_{2}$ at $37^{\circ} \mathrm{C}$.

For gene silencing, lentiviral particles were prepared by transfecting HEK293 cells with LV3 lentiviral vectors encoding specific shRNAs or control shRNAs along with packaging plasmids. The packaged viruses were then used to infect the indicated cells, followed by selection of the infected cells by puromycin (LV3 vector carries the puromycin resistance gene) to establish stable cell lines. Immunoblotting assays were performed to examine the silencing efficiency. For overexpression studies, lentiviral transduction was performed as described above.

\section{Immunoblot Assay}

Total cell lysates and tumor tissue extracts were prepared and subjected to immunoblot assay as described previously (19). Briefly, crude proteins were extracted, resolved by SDSpolyacrylamide gel electrophoresis and transferred onto a PVDF membrane. Membrane were then blocked with 5\% non-fat milk in TBST buffer for $2 \mathrm{~h}$ at $37^{\circ} \mathrm{C}$ and incubated with primary antibodies overnight at $4^{\circ} \mathrm{C}$. After incubation with the appropriate secondary antibody, the immunoblot signal was visualized using ECL reagent.

\section{Cell Proliferation and Colony Formation Assays}

Cell proliferation was assessed by MTT assay. NCI-H358 cells were seeded into 96-well plates at a density of $1 \times 10^{4}$ cells/well in $0.1 \mathrm{~mL}$ medium and cultured for different periods of time as indicated. Then, $20 \mu \mathrm{L}$ MTT reagent $(0.5 \mathrm{mg} / \mathrm{mL}$ in PBS) was added to each well and the plate was incubated for $2 \mathrm{~h}$ before detection. The purple-blue MTT formazan precipitate was dissolved in $100 \mu \mathrm{L}$ DMSO. The absorbance was measured at $490 \mathrm{~nm}$ using a BioTek Epoch Spectrophomometer (BioTek, Winooski, USA). For colony formation, NCI-H358 cells were harvested and pipetted well to become single-cell suspension in RPMI-1640 with $10 \%$ FBS at a concentration of 750 cells $/ \mathrm{mL}$. Cells were then planted into a 6-well plate and incubated at $37^{\circ} \mathrm{C}$ in a humidified $5 \% \mathrm{CO}_{2}$ atmosphere for 2 weeks. After incubation, the cells were fixed with methanol and stained with hexamethyl pararosaniline. Colony formation was then photographed and counted under a phase-contrast microscope.

\section{Cell Migration and Invasion Assays}

Cell migration assay was performed according to the previously described protocol (20). Cells were grown to $100 \%$ confluence on glass slides and a 3-mm-wide scratch was made using a cell scraper. Slides were then incubated at $37^{\circ} \mathrm{C}$ for $24 \mathrm{~h}$ in DMEM containing $10 \mathrm{~g} / \mathrm{L}$ BSA and $1 \%$ FBS. After incubation, the cells were fixed with methanol and stained with hexamethyl pararosaniline. The "wound" was observed under a light microscope. Each sample was randomly selected five fields and photographed. Cell migration was expressed as the migrated distance. For cell invasion assay, a modified Boyden transwell chamber with $8.0 \mathrm{~mm}$ pores was used. The transwell chamber was pre-coated with $100 \mu \mathrm{L}$ of Matrigel $(10 \mathrm{mg} / \mathrm{mL})$ overnight at $4^{\circ} \mathrm{C}$. Cells were seeded $\left(10^{5}\right.$ cells per well $)$ in the upper chamber of the transwell system in DMEM with $0.5 \% \mathrm{FBS}$, and DMEM with $10 \%$ FBS were added to the lower chamber of the transwell system at $500 \mu \mathrm{L}$ per well. Then, cells were incubated at $37^{\circ} \mathrm{C}$ for $6 \mathrm{~h}$. Cells remaining on the upper membrane surface were mechanically removed, and the cells that invaded and migrated to the lower surface of the filter were fixed and stained with $0.5 \%$ crystal violet and observed under the microscope. Each sample was randomly selected five fields and the cells that moved to the lower surface of the filter were photographed and counted.

\section{VEGF ELISA and Tube Formation Assay for Angiogenesis}

NCI-H358 cells were seeded and incubated for $24 \mathrm{~h}$ in culture media. Then, media was removed and cells were incubated in serum-free RPMI-1640 for additional $48 \mathrm{~h}$. Then, conditioned media were obtained. Vascular endothelial growth factor (VEGF) in conditioned media was quantified using VEGF ELISA Kits (Neobioscience Technology, China). To investigate the effect of the conditioned media on the angiogenic activity of EA.hy926 cells in vitro, a tube formation assay was performed according to the protocol described previously (21). EA.hy926 cells were seeded into a 24 -well plate pre-coated with $100 \mu \mathrm{L}$ of $10 \mathrm{mg} / \mathrm{mL}$ 
chilled Matrigel (Corning, Bedford, MA) at a density of $1 \times 10^{5}$ cells/well in $0.5 \mathrm{~mL}$ of culture medium. After $8 \mathrm{~h}$ of incubation, the tube-like structures in five randomly selected microscopic fields in each well were imaged using phase-contrast microscopy and the total lengths were quantified using Image-Pro Plus software 6.0 (Media Cybernetics, Inc., Rockville, MD).

\section{Tumor Xenograft}

Male BALB/c-nu mice, 4-5 weeks old, were purchased from the Beijing HFK Bioscience Co., Ltd. (Beijing, China). All of the mice were bred and housed in a specific pathogen-free environment at Hebei University Laboratory Animal Research Center. All experiments were approved by the Animal Research Ethics Committee of the authors' institution. NCI-H358 cells were cultured in RPMI-1640 supplemented with 10\% FBS. Nude mice were injected subcutaneously with a cell suspension of $0.1 \mathrm{ml}$ in PBS containing $1 \times 10^{6}$ tumor cells into the nude mice. Each experimental group consisted of 6 mice. The challenged mice were monitored for tumor growth, and tumor size was expressed as tumor volume. The volume of the tumors was estimated according the formula: Volume $=1 / 2 \times a \times b^{2}$, where $a$ and $b$ represent the largest and smallest diameters, respectively. Mice were sacrificed at day 15 after injection. The xenograft tumors were dissected, weighted, and photographed.

\section{Statistical Analysis}

Statistical analysis was performed using the SPSS software package (version 19.0, USA). Two-tailed unpaired or paired Student's $t$-tests or one-way analysis of ovariance (ANOVA) with Tukey's multiple comparisons were used according to the number of groups compared. Survival curves were generated using the Kaplan-Meier method, and differences between curves were estimated by the two-sided log-rank test. $P$-values $<0.05$ were considered significant and the level of significance expressed as follows: ${ }^{*} P<0.05$; ${ }^{* *} P<0.01$; ${ }^{* * *} P<0.001$; ${ }^{* * * *} P<0.0001$.

\section{RESULTS}

\section{Elevated OTUD7B Expression Correlates With LUSC and LAD Progression}

OTUD7B, also called Cezanne, was identified as an essential regulator of the $\mathrm{NF}-\kappa \mathrm{B}$ pathway and cancer proliferation, although its physiological function in lung cancer has not been well-defined $(10,11,22)$. The OTUD7B gene was frequently amplified in NSCLC, including LUSC and LAD, according to the TCGA DNA sequencing results (Supplementary Figure S1). To investigate the potential clinical relevance of OTUD7B in lung cancer, we assessed lung cancer tissue samples and matched adjacent normal lung tissue samples from 214 human subjects (143 LUSC cases and 71 LAD cases). IHC analysis revealed
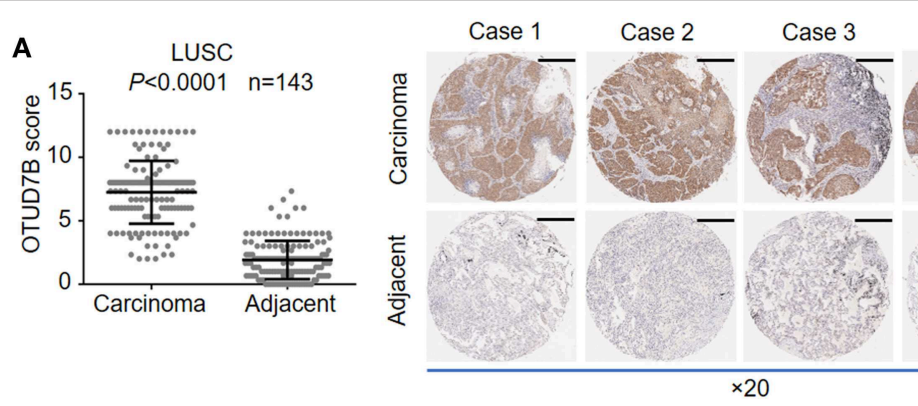

C

B

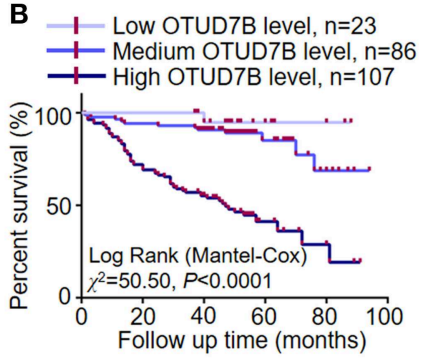

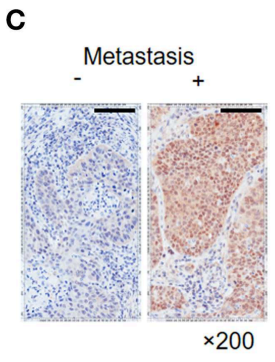

D

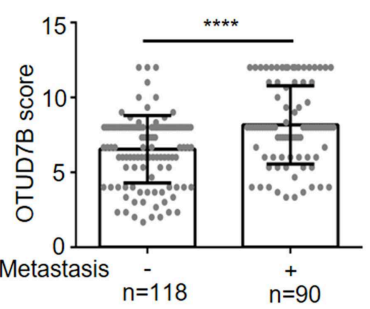

E
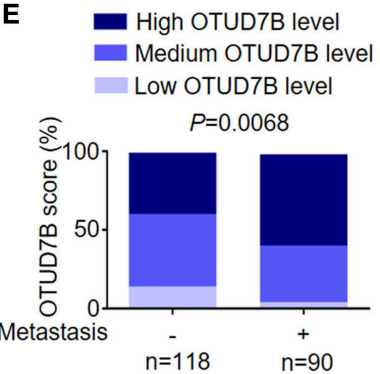

FIGURE 1 | OTUD7B is highly expressed in NSCLCs and correlated with a worse prognosis. (A) OTUD7B expression scores in LUSC and adjacent non-cancer tissues are shown as scatter dot plots. Small horizontal bars indicate the mean \pm s.d. Each dot represents an individual sample. LUSC tissues were compared with matched adjacent non-cancer tissues using paired $t$-test. Right, Representative images of IHC staining of OTUD7B expression from 4 cases are shown. Magnification, $\times 20$ (middle) and ×200 (right); scale bars, $400 \mu \mathrm{m}$. (B) Kaplan-Meier survival curve of high, medium and low OTUD7B level NSCLC. Marks on graph lines represent censored samples. P-value refers to two-sided log-rank tests. (C) Representative images from IHC staining of OTUD7B expression in tumors with or without metastasis (lymph node metastasis and distant metastasis) are shown. Magnification, $\times 200$; scale bars, $100 \mu \mathrm{m}$. (D) Scatter dot plots of OTUD7B expression in the two groups of subjects described in (C) are shown. Data were analyzed using unpaired $t$-test and are shown as mean \pm s.d. ${ }^{* \star \star *} P<0.0001$. (E) The percentage of tumors in the two groups of subjects described in (C). Data were analyzed using Pearson's $\chi^{2}$ test. 
that OTUD7B expression was significantly upregulated in both LUSC (Figure 1A) and LAD (Supplementary Figures S2A-D) tissues compared with adjacent tissues. To better understand the relevance of OTUD7B to cancer a follow-up analysis of patient survival was performed, and the result demonstrated that patients whose tumor had high OTUD7B levels had significantly poorer survival than those with low OTUD7B levels (Figure 1B). Next, we divided the samples into groups based on metastasis (Figures 1C-E) and the AJCC stage (Supplementary Figures S3A-C) to study the correlation of OTUD7B expression with lung cancer development. OTUD7B expression was higher in tumors with lymph node or distance metastasis than that without metastasis (Figures 1C-E).
OTUD7B expression was also lower in low-AJCC stage (AJCC stage I and II) and higher in high-TNM stage (TNM stage III and IV) (Supplementary Figures S3A-C). These data indicate that OTUD7B promotes the metastasis and progression in LUSC and LAD.

\section{OTUD7B Is Associated With Cellular Proliferation, Migration, and Colony Formation in NSCLC Cell Lines}

To address the consequences of OTUD7B-mediated NSCLC progression, we established NSCLC cell line NCI-H358 stably overexpressing OTUD7B (Supplementary Figure S4A)
A

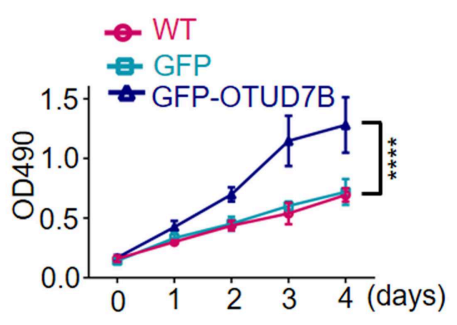

B

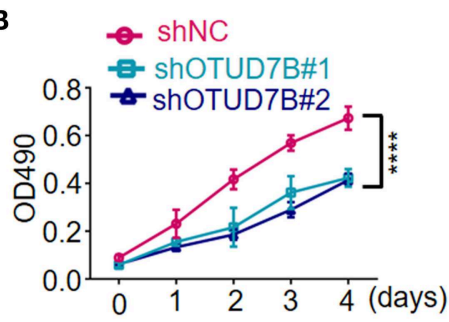

C
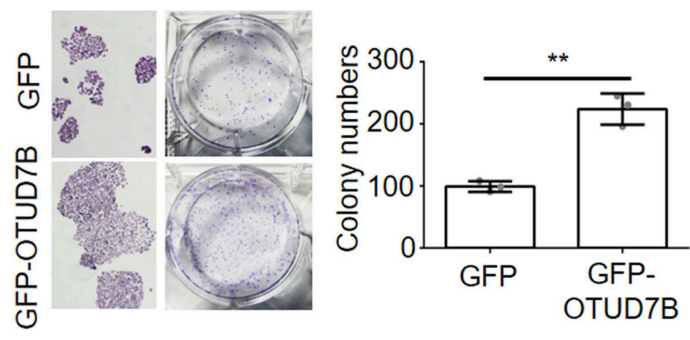

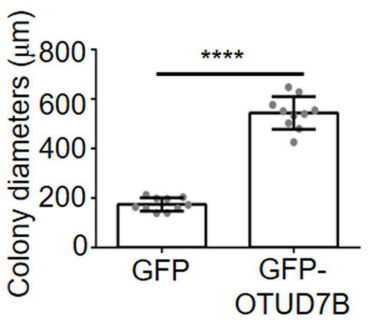

$\mathbf{E}$

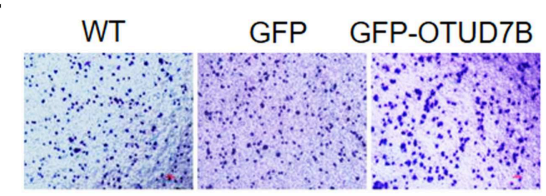

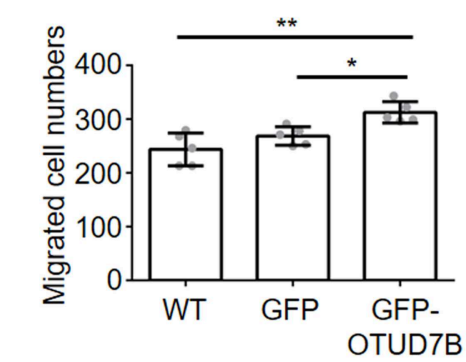

D
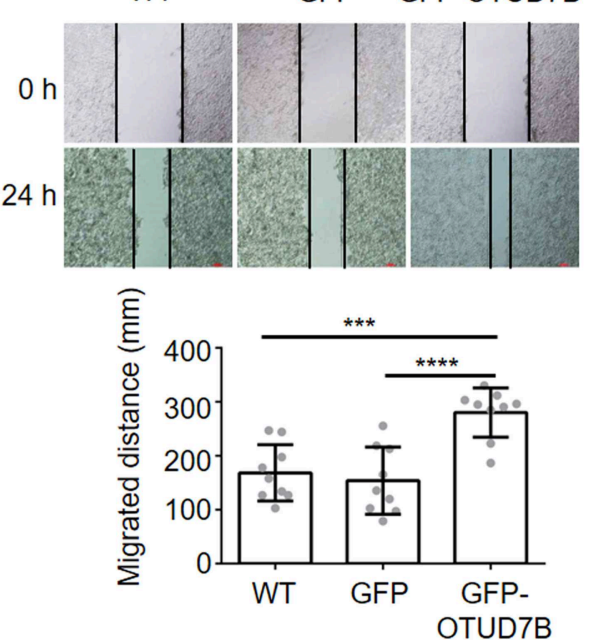

FIGURE 2 | OTUD7B promotes proliferation, migration and colony formation in lung cancer cells. (A,B) NCl-H358 lung cancer cells were transduced with GFP or GFP-OTUD7B (A) or transduced with a non-targeting control shRNA (shNC) or two different OTUD7B-specific shRNAs (B). Cells proliferation was tested using MTT assay and the absorbance was measured at $490 \mathrm{~nm}$. (C) NCl-H358 cells were transduced with GFP or GFP-OTUD7B. Cell proliferation were determined by colony formation analysis. (D,E) Wound healing assay (D) and transwell invasion assay using Boyden chamber (E) in NCl-H358 cells were performed and photographed under a light microscope (magnification, $\times 100$ ). Representative images are shown (upper panels). Data are shown as mean $\pm \mathrm{s} . \mathrm{d}$. ${ }^{\star} P<0.05$, ${ }^{\star \star} P<0.01,{ }^{\star \star \star} P<$ $0.001,{ }^{\star \star \star \star} P<0.0001$. 


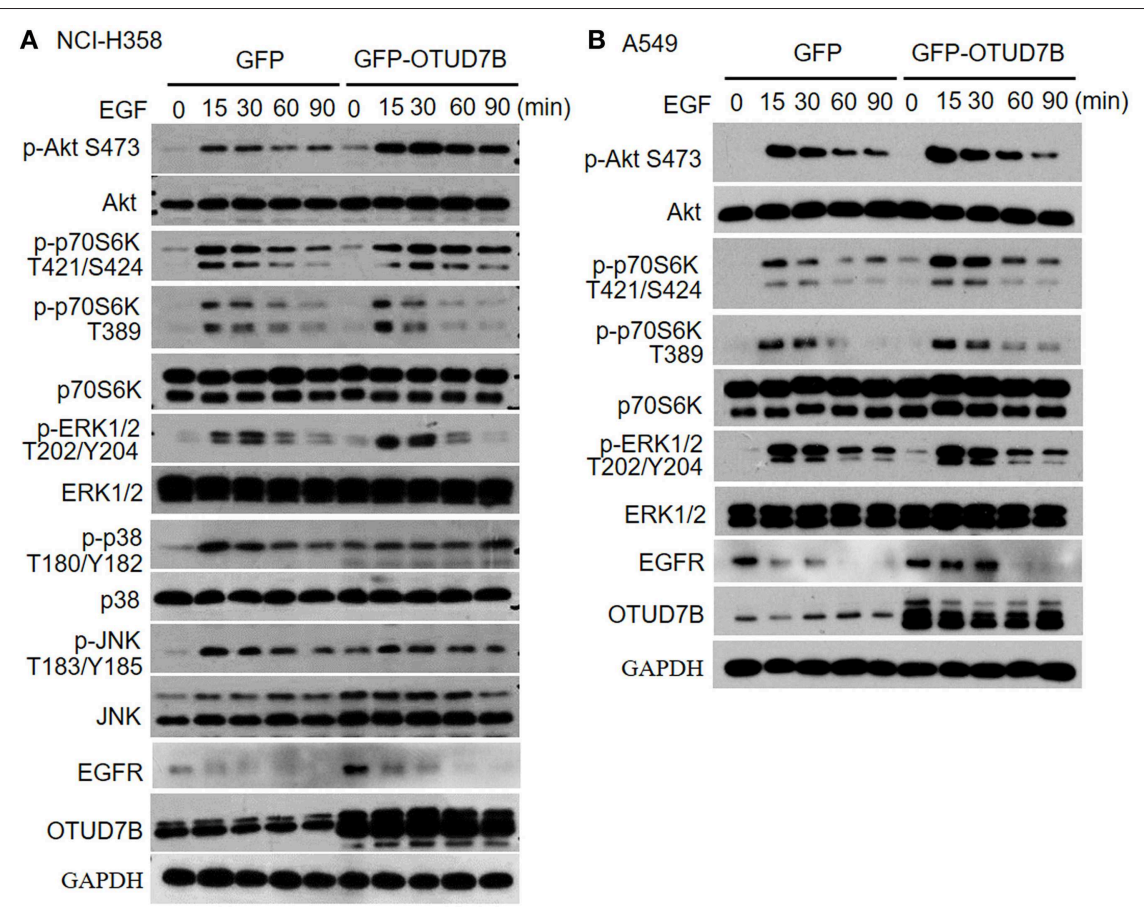

FIGURE 3 | OTUD7B activates Akt and ERK1/2 signals in NSCLC cell lines. NCl-H358 cells (A) or A549 cells (B) transduced with GFP or GFP-OTUD7B were stimulated with EGF $(500 \mathrm{ng} / \mathrm{ml})$ for indicated times and total cell lysates were harvest and subjected to immunoblotting assay.

and performed MTT cell proliferation assay. As expected, overexpression of OTUD7B resulted in an increased proliferation activity in NCI-H358 cells (Figure 2A). Transducing specific shRNA targeting OTUD7B into NCIH358 cells further confirmed that knockdown of endogenous OTUD7B correlates with decreased cell growth (Figure 2B, Supplementary Figure S4B). Furthermore, we examined the effect of OTUD7B on NCI-H358 cell proliferation using a colony formation assay. As shown in Figure 2C, both the colony numbers and colony diameters increased significantly in OTUD7B-overexpressed cells. When endogenous OTUD7B was knocked down, the number and diameter of colony decreased significantly compared with wild type and GFP-overexpression groups (Supplementary Figures S5A-C).

To further investigate the roles of OTUD7B in NSCLC cell biology, cell migration and invasion assays were performed in NCI-H358 and A549 cells. Wound-healing cell migration assay indicated that OTUD7B overexpression significantly promoted cell migration (Figure 2D, Supplementary Figure S6A). Matrigel pre-coated Boyden chamber was used to analyze cell invasion in OTUD7B-overexpressing and control cells. Cells that migrated through the filter were stained and photographed and the results showed that migrated cell numbers significantly increased in OTUD7B-overexpressing cells (Figure 2E, Supplementary Figure S6B).

\section{OTUD7B Promotes p-Akt Level in NSCLC Cell Lines}

Because Akt, ERK1/2, p38, and JNK signaling pathways is crucial for many fundamental cellular processes, we sought to determine whether OTUD7B was involved in these signaling pathways in NCI-H358 and A549 NSCLC cell lines. NCI-H358 cells were stably transduced with GFP or GFP-OTUD7B. Cells were treated with $500 \mathrm{ng} / \mathrm{mL}$ of EGF for 15, 30, 60, and $90 \mathrm{~min}$ as indicated and immunoblotting was performed to examine phosphorylated Akt, ERK1/2, p38, and JNK levels, as well as an Akt downstream kinase, p70S6K pohsphorylation in GFP- and GFP-OTUD7B-overexpressing cells. OTUD7B overexpression markedly increased phosphor-Akt and phospho-p70S6K levels in EGF stimulated NCI-H358 cells (Figure 3A). A slight elevation of phospho-ERK1/2 expression was also detected in OTUD7B-overexpressed NIC-H358 cells (Figure 3A). Moreover, OTUD7B overexpression also facilitated phospho-Akt, ERK1/2, and p70S6K levels in another NSCLC cell line A549 (Figure 3B, Supplementary Figure S4C). EGFR is an important receptor which is well-known as target for personalized therapy (23). We also detected EGFR protein levels in EGF-stimulated GFP- and GFP-OTUD7B-overexpressing cells. As shown in Figures 3A,B, compared with control cells EGFR protein level was significantly higher in OTUD7B-overexpressing cells. Moreover, degradation of EGFR in EGF-stimulated cells was much more rapid in control cells than that in OTUD7B-overexpressing cells. These results indicated that OTUD7B promotes NSCLC cell proliferation through activating EGFR, Akt and ERK1/2 pathways.

\section{VEGF Correlates With OTUD7B Expression in LUSC and LAD}

Recent data indicated that Akt is an important signaling regulator of VEGF production and tumor angiogenesis (24-26). Previous research has also reported that OTUD7B regulates 
A

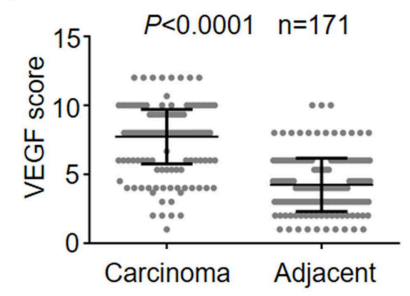

C VEGF

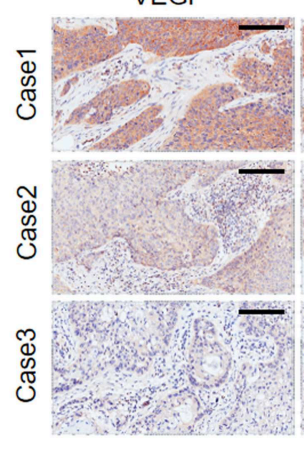

OTUD7B

OTUD7B
B

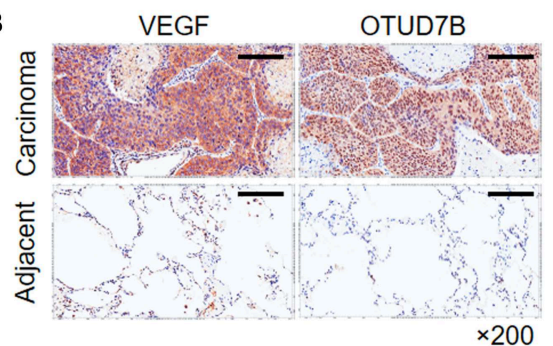

D $\times 200$

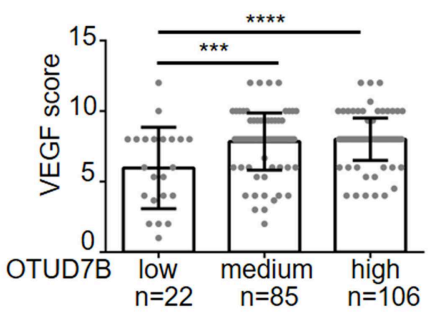

E

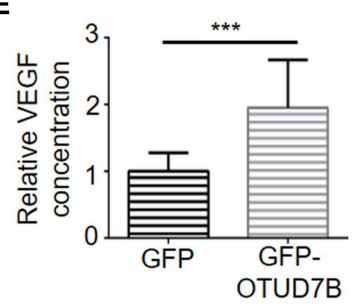

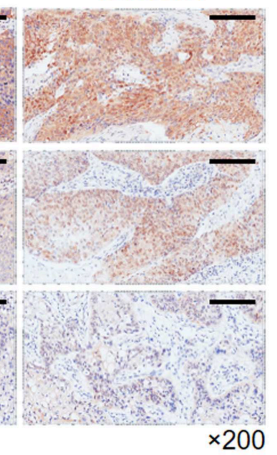

F

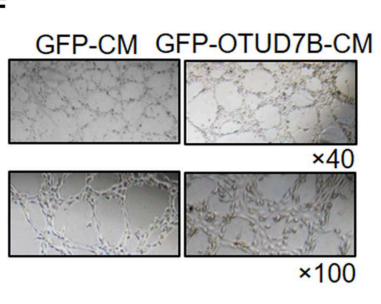

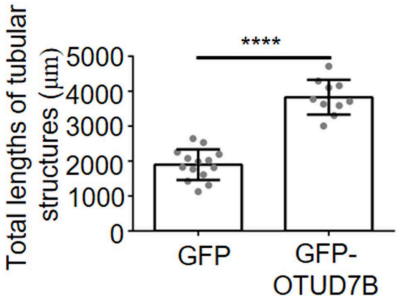

FIGURE 4 | Positive correlation of OTUD7B with VEGF expression and its role in VEGF production and angiogenesis. (A) The expression scores of VEGF $(n=171)$ were compared between NSCLC tumors and matched adjacent normal tissue using paired t-test. Data are shown as mean \pm s.d. (B) Representative images from IHC staining of VEGF in the same NSCLC tumor. Magnification, $\times 200$; Scale bars, $100 \mu \mathrm{m}$. (C) Representative images from IHC staining of VEGF and OTUD7B expression in two serial sections of the same tumor from three cases. Magnification, $\times 200$; Scale bars, $100 \mu \mathrm{m}$. (D) Scatter dot plots of VEGF expression in NSCLC from 213 subjects. The subjects were divided into three groups based on OTUD7B expression scores in the tumors, representing low, medium and high expression of OTUD7B. Data were analyzed by one-way ANOVA and Tukey's multiple comparisons test. (E) Media from GFP- or GFP-OTUD7B-overexpressing NCI-H358 cells was removed, washed in RPMl-1640 with 0.5\% FBS and incubated for an additional $24 \mathrm{~h}$ in RPMl-1640 with 0.5\% FBS. GFP-conditioned media (GFPCM) and GFP-OTUD7B-conditioned media (GFP-OTUD7B-CM) were collected, and VEGF expression were analyzed by ELISA. Absorbance was measured at 450 nm. (F) EA.hy926 endothelial cells were pretreated with GFP-CM and GFP-OTUD7B-CM for $24 \mathrm{~h}$. Subsequently, pretreated EA.hy926 cells were seeded on Matrigel for $8 \mathrm{~h}$ to observe tube formation. Representative photographs are shown (left). Tube lengths were quantitated using IMAGE-PRO PLUS software ( $n=5$ per group). Data are shown as mean \pm s.d. ${ }^{\star \star \star} P<0.001,{ }^{\star \star \star \star} P<0.0001$

hypoxia inducible factors $1 \alpha$ and $2 \alpha$ protein $(13,14)$, which are known effective regulators of VEGF and angiogenesis in cancer. However, little is known about the relation of OTUD7B and VEGF. First, we analyzed the correlation of OTUD7B and VEGF using R2: Genomics Analysis and Visualization Platform (http:// r2.amc.nl). As shown in Supplementary Figure S7, OTUD7B expression was positively associated with VEGF expression ( $r=0.239, p=1.00 \mathrm{e}-06)$. To further determine their relationship, we next examined the expression of VEGF in NSCLC carcinoma tissues and matched normal lung tissues using IHC staining. An analysis of large-scale subjects (171 cases, including 116 cases of LUSC and 55 cases of LAD) demonstrated that VEGF were significantly upregulated in NSCLC carcinoma tissues compared with matched adjacent normal tissues (Figures 4A,B). Importantly, we observed a positive correlation between OTUD7B and VEGF levels among the examined subjects (Figures 4C,D).

To confirm the relevance between OTUD7B and VEGF, we further examined the VEGF concentration in conditional media of NSCLC cell lines that stably overexpress or knockdown OTUD7B. Conditioned media (CM) from NCI-H358 cells stably expressing control-GFP (GFP-CM) or GFP-OTUD7B (GFPOTUD7B-CM) were collected and secreted VEGF levels were examined using ELISA assay. Overexpression of OTUD7B results in higher VEGF secretion in NCI-H358 cells (Figure 4E). When knockdown of endogenous OTUD7B, secreted VEGF levels decreased (Supplementary Figure S8A). Moreover, we examined EGF (another growth factor) secretion in OTUD7B 

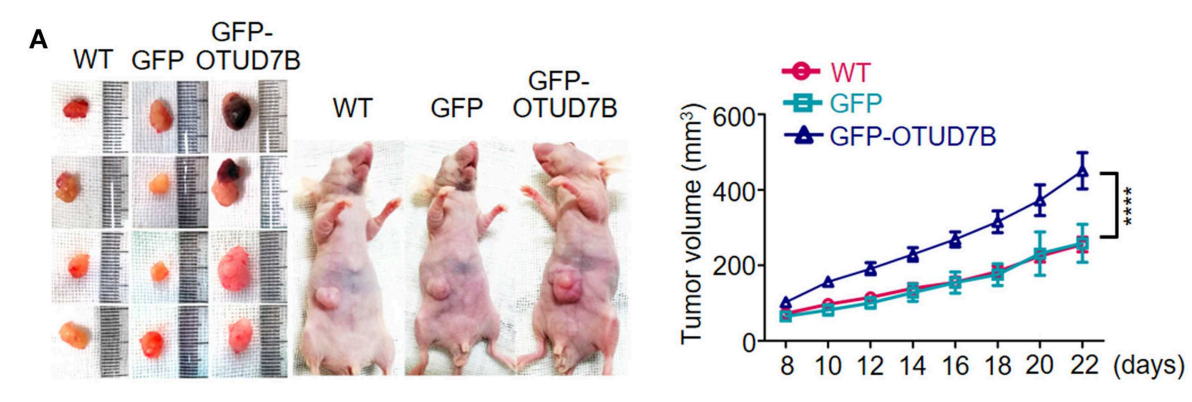

B

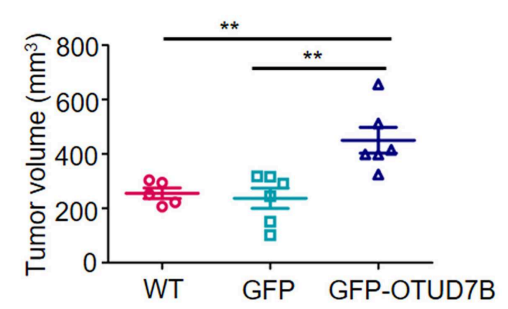

c

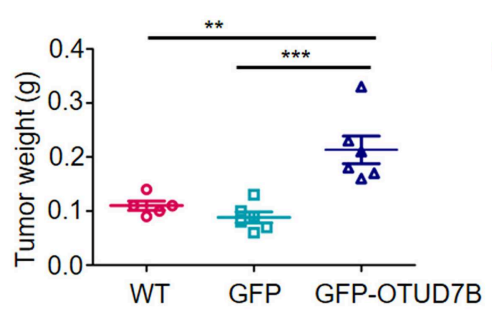

D

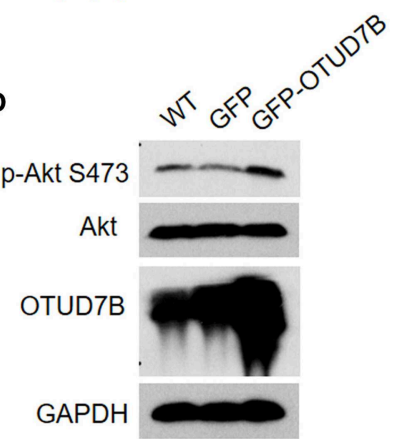

FIGURE 5 | OTUD7B promotes in vivo tumorigenicity of NSCLC via Akt signaling activation. (A) Nude mice were injected s.c. with wild type (WT), GFP- or GFP-OTUD7B-overexpressing NCl-H358 cells at $5 \times 10^{6}$ cells per site. Tumor volume was monitored every 2 days after subcutaneous injection and tumor growth curve is shown (right). Tumor volume was calculated by the formula: $\mathrm{V}=1 / 2 \times a$ (length) $\times b^{2}$ (width). Tumors were harvested from the mice 22 days after subcutaneous injection and photographed (left). (B,C) The volume (B) and the weight (C) of the harvested tumors was measured. (D) Total lysates of tumor tissues were subjected to immunoblotting using anti-p-Akt and anti-Akt antibodies. Data are shown as mean \pm s.e.m. ${ }^{\star \star} P<0.01,{ }^{\star \star *} P<0.001$.

overexpress or knockdown cells. Compared to the control group, there was no significant change of EGF secretion from OTUD7B overexpressing or knockdown NCI-H358 cells (Supplementary Figures S8B,C).

VEGF is an important factor involved in angiogenesis, resulting in tumor progression. Endothelial cells proliferation, migration and tube formation are key processes of angiogenesis which are stimulated by tumor-secreted pro-angiogenic factors, such as VEGF $(27,28)$. We next analyzed whether OTUD7B-induced VEGF secretion from NSCLC cells affect endothelial cell function and promotes angiogenesis. GFPCM and GFP-OTUD7B-CM from NCI-H358 cells were collected and EA.hy926 endothelial cells were pretreated with GFP-CM and GFP-OTUD7B-CM for $24 \mathrm{~h}$, and then seeded on Matrigel to determine tube formation. As shown in Figure 4F, tube length in GFP-OTUD7B-CM-treated EA.hy926 cells was significantly longer than GFP-CMtreated cells. Moreover, CM from control (shNC-CM) and OTUD7B-knockdown NCI-H358 cells (shOTUD7B\#1-CM and shOTUD7B\#2-CM) were collected and CM-induced tube formation analysis showed that tube length in shOTUD7Bs (shOTUD7B\#1 and shOTUD7B\#2)-CM-treated EA.hy926 cells was significantly shorter than shNC-CM-treated cells (Supplementary Figure S8D). Moreover, VEGFR2, an important VEGF receptor, was detected in EA.hy926 endothelial cells and lung cancer cells. The results demonstrated a higher expression of VEGFR2 in ehdothelial cells whereas VEGFR2 levels could hardly be detected in NSCLC cells (Supplementary Figures S9A,B). These results suggest that OTUD7B promotes VEGF production and NSCLC angiogenesis in vitro.

\section{OTUD7B Facilitate Akt Phosphorylation and NSCLC Tumorigenicity in vivo}

The NCI-H358 tumor bearing mouse model was established to further examine the tumorigenic role of OTUD7B. Wild type GFP- or GFP-OTUD7B overexpressing NCI-H358 cells were subcutaneously injected s.c. into the nude mice. The challenged mice were monitored for tumor growth. OTUD7B overexpression dramatically promoted tumorigenicity and tumor growth speed in nude mice (Figures 5A-C). Furthermore, Akt phosphorylation at S473 site was increased in OTUD7Boverexpressing tumors compared to the wild type and GFP control tumors (Figure 5D). To further confirm the role of OTUD7B in lung cancer progression, OTUD7B stably knockdown NCI-H358 cells were used to generate xenograft nude mouse model. Knockdown of OTUD7B in NCI-H358 cells significantly decreased Akt phosphorylation at S473 and inhibited tumor growth (Supplementary Figures S10A-D). These results suggest that OTUD7B is required for lung tumor progression at least partially by facilitating activation of Akt signaling.

\section{DISCUSSION}

Lung and bronchus cancer continues to be the leading cause of cancer mortality worldwide in both males (76,650 estimated deaths in 2019) and females (66,020 deaths) for more than 25 
years (29). In spite of the rapid development of diagnostic and therapeutic technologies for NSCLC, its outcome remains poor, which may due to the lack of specific targets at the early stage $(2,30)$. Recent studies have demonstrated OTUD7B as a gene highly expressed in lung adenocarcinoma tissues $(12,18)$. In the present study, OTUD7B was revealed to overexpressed within LAD and LUSC tissues compared with adjacent noncancerous tissues. High expression of OTUD7B predicts metastasis and poor survival in NSCLC patients, which suggested potential role of OTUD7B in lung cancer initiation and progression.

In recent years, increasing evidence indicated the vital role of ubiquitinases and DUBs in the progression of NSCLC $(3,4$, $31,32)$. OTUD7B acts as a multifunctional DUB by targeting Lys11- (33, 34), Lys48- (8, 9), and Lys63-linked (12) ubiquitin chains. The essential role of OTUD7B in the activation of inflammation $(7,9,35)$ and NF- $\mathrm{NB}$ pathway $(8,11)$ has been extensively investigated. However, the role of OTUD7B in tumor growth and metastasis signals is still not clear. We demonstrated OTUD7B expression level affects cell growth and colony formation in NCI-H358 cells. Phosphorylation of Akt, as well its downstream kinase, p70S6K, and ERK1/2 was significantly elevated by OTUD7B overexpression in EGFtreated NSCLC cell lines, NCI-H358 and A549. These data suggested that OTUD7B functions as an oncogene via facilitating Akt activation.

Angiogenesis is the formation of new blood vessels from pre-existing vasculature, which is essential for solid tumor growth and metastasis. The VEGF signal pathway has been well-characterized in lung cancer pathogenesis and anti-VEGF treatment has been considered one of the most effective strategies against advanced NSCLC. Recent evidence demonstrated Akt participates in VEGF production $(24,36)$. OTUD7B promotes EGF-stimulated Akt activation in NSCLC cell lines, however the correlation of OTUD7B and VEGF expression is still unclear. An IHC analysis of 171 NSCLC patient specimens revealed that VEGF was significantly upregulated in cancer tissues compared with adjacent tissues. Patients whose NSCLC featured high OTUD7B expression had high a VEGF level and especially poor prognosis. Further studies in NCI-H358 cells suggested that OTUD7B promoted VEGF secretion and facilitated endothelial cell tube formation in vitro. In addition, OTUD7B was demonstrated to promote tumor growth in NCI-H358 lung cancer xenografts. These findings may have significant implicants for the treatment of NSCLC via targeting OTUD7B.

\section{REFERENCES}

1. Chen M, Liu X, Du J, Wang XJ, Xia L. Differentiated regulation of immuneresponse related genes between LUAD and LUSC subtypes of lung cancers. Oncotarget. (2017) 8:133-44. doi: 10.18632/oncotarget.13346

2. Carmichael JA, Wing-San Mak D, O'Brien M. A review of recent advances in the treatment of elderly and poor performance NSCLC. Cancers. (2018) 10:236. doi: $10.3390 /$ cancers 10070236

3. Wang S, Xu L, Che X, Li C, Xu L, Hou K, et al. E3 ubiquitin ligases Cbl-b and cCbl downregulate PD-L1 in EGFR wild-type non-small cell lung cancer. FEBS Lett. (2018) 592:621-30. doi: 10.1002/1873-3468.12985
Taken together, this study identified OTUD7B as an independent predictive factor for the prognosis of LUSC and LAD. OTUD7B promotes lung cancer cell proliferation and migration via Akt/VEGF signal pathway. Strategies to silencing OTUD7B in carcinoma tissues may inform the development of novel therapies to fight against LUSC and LAD.

\section{DATA AVAILABILITY}

The datasets generated for this study are available on request to the corresponding author.

\section{ETHICS STATEMENT}

This study was carried out in accordance with the recommendations of Institutional Review Board of Hebei University Affiliated Hospital with written informed consent from all subjects. All subjects gave written informed consent in accordance with the Declaration of Helsinki. The protocol was approved by the Institutional Review Board of Hebei University Affiliated Hospital. This study was carried out in accordance with the recommendations of the Animal Research Ethics Committee of Hebei University. The protocol was approved by the Animal Research Ethics Committee of Hebei University.

\section{AUTHOR CONTRIBUTIONS}

DL, YS, SQ, WL, LZ, NC, and YuW performed the experiments. DL, YS, YaW, and NC collected and analyzed data. SZ and JS conceived and designed the project. DL, SZ, and JS wrote the manuscript.

\section{FUNDING}

This work was supported by the National Natural Science Foundation of China (Grant No. 31301143 and 81572557), the Natural Science Foundation of Hebei Province (Grant No. H2019201259), and the Hebei Youth Topnotch Talent Support Program.

\section{SUPPLEMENTARY MATERIAL}

The Supplementary Material for this article can be found online at: https://www.frontiersin.org/articles/10.3389/fonc. 2019.00862/full\#supplementary-material

4. Gu J, Mao W, Ren W, Xu F, Zhu Q, Lu C, et al. Ubiquitin-protein ligase E3C maintains non-small-cell lung cancer stemness by targeting AHNAKp53 complex. Cancer Lett. (2019) 443:125-34. doi: 10.1016/j.canlet.2018. 11.029

5. Zhou B, Zuo Y, Li B, Wang H, Liu H, Wang X, et al. Deubiquitinase inhibition of $19 \mathrm{~S}$ regulatory particles by 4 -arylidene curcumin analog AC17 causes NFkappaB inhibition and p53 reactivation in human lung cancer cells. Mol Cancer Ther. (2013) 12:1381-92. doi: 10.1158/1535-7163.MCT-12-1057

6. Zhang L, Xu B, Qiang Y, Huang H, Wang C, Li D, et al. Overexpression of deubiquitinating enzyme USP28 promoted non-small cell lung cancer growth. J Cell Mol Med. (2015) 19:799-805. doi: 10.1111/jcmm.12426 
7. Enesa K, Zakkar M, Chaudhury H, Luong A, Rawlinson L, Mason JC, et al. NF-kappaB suppression by the deubiquitinating enzyme Cezanne: a novel negative feedback loop in pro-inflammatory signaling. J Biol Chem. (2008) 283:7036-45. doi: 10.1074/jbc.M708690200

8. Hu H, Brittain GC, Chang JH, Puebla-Osorio N, Jin J, Zal A, et al. OTUD7B controls non-canonical NF-kappaB activation through deubiquitination of TRAF3. Nature. (2013) 494:371-4. doi: 10.1038/nature11831

9. Luong A, Fragiadaki M, Smith J, Boyle J, Lutz J, Dean JL, et al. Cezanne regulates inflammatory responses to hypoxia in endothelial cells by targeting TRAF6 for deubiquitination. Circ Res. (2013) 112:158391. doi: 10.1161/CIRCRESAHA.111.300119

10. Chiu HW, Lin HY, Tseng IJ, Lin YF. OTUD7B upregulation predicts a poor response to paclitaxel in patients with triple-negative breast cancer. Oncotarget. (2018) 9:553-65. doi: 10.18632/oncotarget.23074

11. Ji Y, Cao L, Zeng L, Zhang Z, Xiao Q, Guan P, et al. The N-terminal ubiquitinassociated domain of Cezanne is crucial for its function to suppress NFkappaB pathway. J Cell Biochem. (2018) 119:1979-91. doi: 10.1002/jcb.26359

12. Wang B, Jie Z, Joo D, Ordureau A, Liu P, Gan W, et al. TRAF2 and OTUD7B govern a ubiquitin-dependent switch that regulates mTORC2 signalling. Nature. (2017) 545:365-9. doi: 10.1038/nature22344

13. Moniz S, Bandarra D, Biddlestone J, Campbell KJ, Komander D, Bremm A, et al. Cezanne regulates E2F1-dependent HIF2alpha expression. J Cell Sci. (2015) 128:3082-93. doi: 10.1242/jcs.168864

14. Bremm A, Moniz S, Mader J, Rocha S, Komander D. Cezanne (OTUD7B) regulates HIF-1alpha homeostasis in a proteasome-independent manner. EMBO Rep. (2014) 15:1268-77. doi: 10.15252/embr.201438850

15. Pareja F, Ferraro DA, Rubin C, Cohen-Dvashi H, Zhang F, Aulmann S, et al. Deubiquitination of EGFR by Cezanne-1 contributes to cancer progression. Oncogene. (2012) 31:4599-608. doi: 10.1038/onc.2011.587

16. Wang JH, Wei W, Guo ZX, Shi M, Guo RP. Decreased Cezanne expression is associated with the progression and poor prognosis in hepatocellular carcinoma. J Transl Med. (2015) 13:41. doi: 10.1186/s12967-015-0396-1

17. Zhang B, Wang H, Yang L, Zhang Y, Wang P, Huang G, et al. OTUD7B and NIK expression in non-small cell lung cancer: association with clinicopathological features and prognostic implications. Pathol Res Pract. (2016) 212:893-8. doi: 10.1016/j.prp.2016.07.011

18. Pang Z, Cui L, Ding N, Zhu L, Qu X, Dong W, et al. Expressions of insulin-like growth factor receptor-1 and cezanne-1 in lung adenocarcinoma. Med Oncol. (2017) 34:78. doi: 10.1007/s12032-017-0934-1

19. Shi JH, Cui NP, Wang S, Zhao MZ, Wang B, Wang YN, et al. Overexpression of YB1 C-terminal domain inhibits proliferation, angiogenesis and tumorigenicity in a SK-BR-3 breast cancer xenograft mouse model. FEBS Open Biol. (2016) 6:33-42. doi: 10.1002/2211-5463.12004

20. Shi JH, Zheng B, Li YH, Sun Y, Han AL, Zhang XH, et al. Novel insight into Y-box binding protein 1 in the regulation of vascular smooth muscle cell proliferation through targeting GC box-dependent genes. FEBS Lett. (2013) 587:1326-32. doi: 10.1016/j.febslet.2013.02.047

21. Wang W, Wang HJ, Wang B, Li Y, Qin Y, Zheng LS, et al. The role of the $\mathrm{Y}$ box binding protein $1 \mathrm{C}$-terminal domain in vascular endothelial cell proliferation, apoptosis, and angiogenesis. DNA Cell Biol. (2016) 35:2432. doi: 10.1089/dna.2015.2908

22. Bonacci T, Suzuki A, Grant GD, Stanley N, Cook JG, Brown NG, et al. Cezanne/OTUD7B is a cell cycle-regulated deubiquitinase that antagonizes the degradation of APC/C substrates. EMBO J. (2018) 37:e98701. doi: 10.15252/embj.201798701

23. Reckamp KL, Frankel PH, Ruel N, Macke PC, Gitlitz BJ, Li T, et al. Phase II trial of cabozantinib plus erlotinib in patients with advanced epidermal growth factor Receptor (EGFR)-mutant non-small cell lung cancer with progressive disease on epidermal growth factor receptor tyrosine kinase inhibitor therapy: a california cancer consortium phase II trial (NCI 9303). Front Oncol. (2019) 9:132. doi: 10.3389/fonc.2019.00132

24. Zhang J, Su H, Li Q, Li J, Zhao Q. Genistein decreases A549 cell viability via inhibition of the PI3K/AKT/HIF1alpha/VEGF and NFkappaB/COX2 signaling pathways. Mol Med Rep. (2017) 15:2296-302. doi: 10.3892/mmr.2017.6260

25. Zhang W, Xiong Z, Wei T, Li Q, Tan Y, Ling L, et al. Nuclear factor 90 promotes angiogenesis by regulating HIF-1alpha/VEGF-A expression through the PI3K/Akt signaling pathway in human cervical cancer. Cell Death Dis. (2018) 9:276. doi: 10.1038/s41419-018-0334-2

26. Zhao X, Yu FQ, Huang XJ, Xu BY, Li YL, Zhao XY, et al. Azithromycin influences airway remodeling in asthma via the PI3K/Akt/MTOR/HIF1alpha/VEGF pathway. J Biol Regul Homeost Agents. (2018) 32:1079-88.

27. Bremnes RM, Camps C, Sirera R. Angiogenesis in non-small cell lung cancer: the prognostic impact of neoangiogenesis and the cytokines VEGF and bFGF in tumors and blood. Lung Cancer. (2006) 51:14358. doi: 10.1016/j.lungcan.2005.09.005

28. Hida K, Maishi N, Torii C, Hida Y. Tumor angiogenesischaracteristics of tumor endothelial cells. Int J Clin Oncol. (2016) 21:206-12. doi: 10.1007/s10147-016-0957-1

29. Siegel RL, Miller KD, Jemal A. Cancer statistics, 2019. CA Cancer J Clin. (2019) 69:7-34. doi: 10.3322/caac.21551

30. Camidge DR, Doebele RC, Kerr KM. Comparing and contrasting predictive biomarkers for immunotherapy and targeted therapy of NSCLC. Nat Rev Clin Oncol. (2019) 16:341-55. doi: 10.1038/s41571-019-0173-9

31. McFarlane C, McFarlane S, Paul I, Arthur K, Scheaff M, Kerr K, et al. The deubiquitinating enzyme USP17 is associated with non-small cell lung cancer (NSCLC) recurrence and metastasis. Oncotarget. (2013) 4:183643. doi: 10.18632/oncotarget.1282

32. Wu N, Liu C, Bai C, Han YP, Cho WC, Li Q. Over-expression of deubiquitinating enzyme USP14 in lung adenocarcinoma promotes proliferation through the accumulation of beta-catenin. Int J Mol Sci. (2013) 14:10749-60. doi: 10.3390/ijms140610749

33. Bremm A, Freund SM, Komander D. Lys11-linked ubiquitin chains adopt compact conformations and are preferentially hydrolyzed by the deubiquitinase Cezanne. Nat Struct Mol Biol. (2010) 17:939-47. doi: 10.1038/nsmb.1873

34. Mevissen TET, Kulathu Y, Mulder MPC, Geurink PP, Maslen SL, Gersch M, et al. Molecular basis of Lys11-polyubiquitin specificity in the deubiquitinase Cezanne. Nature. (2016) 538:402-5. doi: 10.1038/nature19836

35. Abe J, Berk BC. Cezanne paints inflammation by regulating ubiquitination. Circ Res. (2013) 112:1526-8. doi: 10.1161/CIRCRESAHA.113.301518

36. Di Y, Chen XL. Inhibition of LY294002 in retinal neovascularization via down-regulation the PI3K/AKT-VEGF pathway in vivo and in vitro. Int $J$ Ophthalmol. (2018) 11:1284-9. doi: 10.18240/ijo.2018.08.06

Conflict of Interest Statement: The authors declare that the research was conducted in the absence of any commercial or financial relationships that could be construed as a potential conflict of interest.

Copyright (c) 2019 Lin, Shen, Qiao, Liu, Zheng, Wang, Cui, Wang, Zhao and Shi. This is an open-access article distributed under the terms of the Creative Commons Attribution License (CC BY). The use, distribution or reproduction in other forums is permitted, provided the original author(s) and the copyright owner(s) are credited and that the original publication in this journal is cited, in accordance with accepted academic practice. No use, distribution or reproduction is permitted which does not comply with these terms. 